\title{
THE STOCHASTIC WICK-TYPE BURGERS EQUATION
}

\author{
H. Holden ${ }^{1)}$, T. Lindstrøm ${ }^{2)}$, B. Ø Øksendal ${ }^{3)}$, J. Ubøe ${ }^{4)}$ and T.-S. Zhang ${ }^{4)}$
}

\begin{abstract}
We study the multidimensional stochastic (Wick-type) Burgers equation

$$
\left\{\begin{array}{cl}
\frac{\partial u_{k}}{\partial t}+\lambda \sum_{j=1}^{n} u_{j} \diamond \frac{\partial u_{k}}{\partial x_{j}} \quad & =\nu \Delta u_{k}+w_{k}(t, x) ; t>0, x \in \mathbf{R}^{n} \\
& u_{k}(0, x)=g_{k}(x) ; 1 \leq k \leq n
\end{array}\right.
$$

where $\diamond$ denotes the Wick product, $\lambda$ and $\nu$ are constants $(\nu>0, \lambda \neq 0), \Delta$ denotes the Laplacian and $\left\{w_{k}(t, x)\right\}_{k=1}^{n}$ are $(n+1)$-parameter stochastic processes (noise). We prove an existence and uniqueness result for the solution $u(t, x)=\left\{u_{k}(t, x)\right\}_{k=1}^{n}$, regarded as an $(n+1)$-parameter stochastic process with values in the Kondratiev space $(\mathcal{S})^{-1}$ of stochastic distributions.
\end{abstract}

\section{CONTENTS}

$\S 0$. INTRODUCTION

$\S 1$. WHITE NOISE, WICK PRODUCTS AND STOCHASTIC DISTRIBUTIONS

$\S 2$. FROM THE STOCHASTIC BURGERS EQUATION TO THE STOCHASTIC HEAT EQUATION

$\S 3 .(\mathcal{S})^{-1}$-SOLUTION OF THE STOCHASTIC HEAT EQUATION

$\S 4$. FROM THE STOCHASTIC HEAT EQUATION TO THE STOCHASTIC BURGERS EQUATION

§5. AN EXISTENCE AND UNIQUENESS RESULT REFERENCES

1.) Dept. of Mathematical Sciences, Norwegian Institute of Technology, University of Trondheim, N-7034 Trondheim, Norway

2) Dept. of Mathematics, University of Oslo, Box 1053, Blindern, N-0316 Oslo, Norway

3) Dept. of Mathematics/VISTA, University of Oslo, Box 1053, Blindern, N-0316 Oslo, Norway

4) Dept. of Mathematics, National College of Safety Engineering, Skåregt. 103, N-5500 Haugesund, Norway. 


\section{§0. INTRODUCTION}

The purpose of this paper is to continue the work done in [HLØUZ 2] regarding the stochastic multidimensional Burgers equation in $\left\{u_{k}(t, x)\right\}_{k=1}^{n}$ :

$$
\left\{\begin{aligned}
\frac{\partial u_{k}}{\partial t}+\lambda \sum_{j=1}^{n} u_{j} \diamond \frac{\partial u_{k}}{\partial x_{j}} & =\nu \Delta u_{k}+w_{k}(t, x) ; t>0, x \in \mathbf{R}^{n} \\
& u_{k}(0, x)=g_{k}(x) ; 1 \leq k \leq n
\end{aligned}\right.
$$

where $\diamond$ denotes the Wick product, $\lambda$ and $\nu$ are constants $(\nu>0, \lambda \neq 0), \Delta$ denotes the Laplacian and $w_{k}(t, x)=w_{k}(t, x)$ are $(n+1)$-parameter generalized stochastic processes; $1 \leq k \leq n$. (See details below). The use of the Wick product corresponds to an Ito/Skorohod interpretation of the equation (see e.g. [B1], [LØU 2] and also [HLØUZ 3]). We may regard $u=\left(u_{1}, \cdots, u_{n}\right)$ as the velocity field of a vorticity free fluid with viscosity $\nu$, being exposed to the stochastic force $w=\left(w_{1}, \cdots, w_{n}\right)$.

In [HL ØUZ 2] the following was proved: (For definition of functional processes etc. see $\S 1)$. (Here - and in the following - all gradients are taken w.r.t. $x$ ).

A. Let $N=N(\phi, t, x, \omega)$ be a functional process and define $w=-\nabla N$. Assume that (0.1) has a solution of the form

$$
u=-\nabla X
$$

for some functional process $X=X(\phi, t, x, \omega)$. Moreover, assume that the functional processes

$$
Y:=\operatorname{Exp}\left(\frac{\lambda}{2 \nu} X\right)
$$

as well as $Y \diamond X$ and $Y \diamond X^{\diamond 2}$ exist in $L^{p}(\mu)$ for some $p \geq 1$. Then $Y$ solves the stochastic heat equation

$$
\left\{\begin{array}{l}
\frac{\partial Y}{\partial t}=\nu \Delta Y+\frac{\lambda}{2 \nu} Y \diamond(N+C) ; \quad t>0, x \in \mathbf{R}^{n} \\
Y(0, x)=\operatorname{Exp}\left(\frac{\lambda}{2 \nu} X(0, x)\right)
\end{array}\right.
$$

for some $C(t, \omega)$ not depending on $x$. (For simplicity we have written $Y(t, x)$ for $Y(\phi, x, x, \omega)$ etc. $)$

Furthermore, we proved the following:

B. Let either $N=W(\phi, t, x, \omega)((n+1)$-parameter white noise) or $N=\operatorname{Exp} W(\phi, t, x, \omega)$ $((n+1)$-parameter positive noise), and assume that both $f(x):=Y(0, x)$ and $C(t, \omega)=$ $C(t)$ are bounded and deterministic (do not depend on $\omega$ ).

Then (0.3) has a unique $L^{2}(\mu)$ (respectively $L^{1}(\mu)$ ) functional process solution $Y(t, x)$ given by

$$
Y(t, x)=\hat{E}^{x}\left[f\left(b_{\alpha t}\right) \operatorname{Exp}\left(\int_{0}^{t} H\left(s, b_{\alpha s}\right) d s\right)\right]
$$


where $\alpha=\sqrt{2 \nu},\left(b_{t}, \hat{P}^{x}\right)$ is standard Brownian motion in $\mathbf{R}^{n}\left(\hat{E}^{x}\right.$ denotes expectation w.r.t. $\hat{P}^{x}$ ) and

$$
H(t, x)=H(t, x, \omega)=\frac{\lambda}{2 \nu}(N(\phi, t, x, \omega)+C(t))
$$

Thus we see that if $u$ is a gradient solution of (0.1) then we can use it to construct an explicit solution of the stochastic heat equation (0.3). Moreover, by other methods the unique solution of (0.3) can be found explicitly. In particular, this proves that under the given assumptions there exists at most one functional process solution (of gradient form) of the stochastic Burgers equation (0.1).

The purpose of the present paper is to complete the analysis by proving that one can reverse the Wick-Cole-Hopf transformation (0.2) to construct a solution of the stochastic Burgers equation from the stochastic heat equation and hence obtain a uniqueness and existence result for equation (0.1). In order to accomplish this we consider processes and operations in the Kondratiev space $(\mathcal{S})^{-1}$ of stochastic distributions. This space has already found several applications in stochastic partial (and ordinary) differential equations. See e.g. [B 2], [HL $\varnothing U Z$ Z] and [ $\varnothing]$. For the stochastic Burgers equation this approach has the following advantages:

a) The transformation from the stochastic Burgers equation to the stochastic heat equation can be performed with fewer assumptions than given above if done in the space $(\mathcal{S})^{-1}$. (See Theorem 2.1 ).

b) The stochastic heat equation can be solved explicitly in $(\mathcal{S})^{-1}$ for a general $(\mathcal{S})^{-1}$ potential (Theorem 3.1).

c) Most importantly, in $(\mathcal{S})^{-1}$ one can also construct the converse transformation from the stochastic heat equation to the stochastic Burgers equation. (See Theorem 4.1).

By combining a), b) and c) we obtain a uniqueness and existence result (Theorem 5.1) for the stochastic Burgers equation (0.1). Moreover, we obtain this under weaker assumptions than what was needed for the uniqueness result in [HLØUZ 2].

One-dimensional Burgers equations with ordinary product instead of Wick product have been studied in [BCJ-L], [DDT] and [DG].

\section{$\S 1$. WHITE NOISE, WICK PRODUCTS AND STOCHASTIC DISTRIBU- TIONS}

Here we briefly recall some of the basic definitions and results that we need from white noise calculus. For more information the reader is referred to [HKPS] and [KLS].

In the following we fix the parameter dimension $d$ and let $\mathcal{S}=\mathcal{S}\left(\mathbf{R}^{d}\right)$ denote the Schwartz space of rapidly decreasing smooth $\left(C^{\infty}\right)$ functions on $\mathbf{R}^{d}$. The dual $\mathcal{S}^{\prime}=\mathcal{S}^{\prime}\left(\mathbf{R}^{d}\right)$ is 
the space of tempered distributions. By the Bochner-Minlos theorem [GV] there exists a probability measure $\mu$ on the Borel subsets $\mathcal{B}$ of $\mathcal{S}^{\prime}$ with the property that

$$
\int_{\mathcal{S}^{\prime}} e^{i\langle\omega, \phi\rangle} d \mu(\omega)=e^{-\frac{1}{2}\|\phi\|^{2}} ; \forall \phi \in \mathcal{S}
$$

where $\langle\omega, \phi\rangle$ denotes the action of $\omega \in \mathcal{S}^{\prime}$ on $\phi \in \mathcal{S}$ and $\|\phi\|^{2}=\int_{\mathbf{R}^{d}}|\phi(x)|^{2} d x$. The triple $\left(\mathcal{S}^{\prime}, \mathcal{B}, \mu\right)$ is called the white noise probability space.

The white noise process is the map $W: \mathcal{S} \times \mathcal{S}^{\prime} \rightarrow \mathbf{R}$ defined by

$$
W(\phi, \omega)=W_{\phi}(\omega)=\langle\omega, \phi\rangle ; \quad \omega \in \mathcal{S}^{\prime}, \phi \in \mathcal{S}
$$

Expressed in terms of Ito integrals with respect to $d$-parameter Brownian motion $B$ we have

$$
W_{\phi}(\omega)=\int_{\mathbf{R}^{d}} \phi(x) d B_{x}(\omega) \quad ; \phi \in \mathcal{S} .
$$

The Hermite polynomials are defined by

$$
h_{n}(x)=(-1)^{n} e^{\frac{x^{2}}{2}} \frac{d^{n}}{d x^{n}}\left(e^{-\frac{x^{2}}{2}}\right) ; \quad n=0,1,2, \cdots
$$

and the Hermite functions are defined by

$$
\xi_{n}(x)=\pi^{-\frac{1}{4}}((n-1) !)^{-\frac{1}{2}} e^{-\frac{x^{2}}{2}} h_{n-1}(\sqrt{2} x) \quad ; \quad n \geq 1
$$

In the following we let $\left\{e_{1}, e_{2}, \cdots\right\} \subset \mathcal{S}$ denote a fixed orthonormal basis for $L^{2}\left(\mathbf{R}^{d}\right)$. For many purposes the basis can be arbitrary, but for us it is convenient to assume that the $e_{n}$ 's are obtained by taking tensor products of $\xi_{k}(x)$. Define

$$
\theta_{j}(\omega):=W_{e_{j}}(\omega)=\int_{\mathbf{R}^{d}} e_{j}(x) d B_{x}(\omega) \quad ; \quad j=1,2, \cdots
$$

If $\alpha=\left(\alpha_{1}, \cdots, \alpha_{m}\right)$ is a multi-index of non-negative integers we put

$$
H_{\alpha}(\omega)=\prod_{j=1}^{m} h_{\alpha_{j}}\left(\theta_{j}\right)
$$

The Wiener-Ito chaos expansion theorem says that any $X \in L^{2}(\mu)$ can be (uniquely) written

$$
X(\omega)=\sum_{\alpha} c_{\alpha} H_{\alpha}(\omega)
$$


Moreover,

$$
\|X\|_{L^{2}(\mu)}^{2}=\sum_{\alpha} \alpha ! c_{\alpha}^{2} \quad \text { where } \quad \alpha !=\alpha_{1} ! \alpha_{2} ! \cdots \alpha_{m} !
$$

The Hida test function space $(\mathcal{S})$ and the Hida distribution space $(\mathcal{S})^{*}$ can be given the following characterization, due to T.-S. Zhang [Z]:

\section{THEOREM $1.1([\mathrm{Z}])$}

Part a): A function $f=\sum_{\alpha} c_{\alpha} H_{\alpha} \in L^{2}(\mu)$ belongs to $(\mathcal{S})$ if and only if

$$
\sup _{\alpha} c_{\alpha}^{2} \alpha !(2 \mathbf{N})^{\alpha k}<\infty \quad \forall k<\infty
$$

where

$$
(2 \mathrm{~N})^{\alpha}:=\prod_{j=1}^{m}\left(2^{d} \beta_{1}^{(j)} \cdots \beta_{d}^{(j)}\right)^{\alpha_{j}} \quad \text { if } \alpha=\left(\alpha_{1}, \cdots, \alpha_{m}\right)
$$

Here $\beta^{(j)}=\left(\beta_{1}^{(j)}, \cdots, \beta_{d}^{(j)}\right)$ is multi-index $\mathrm{nr} . j$ in the fixed ordering of all $d$-dimensional multi-indices $\beta=\left(\beta_{1}, \cdots, \beta_{d}\right)$, related to the basis $\left\{e_{j}\right\}$ by

$$
e_{j}=\xi_{\beta_{1}^{(j)}} \otimes \cdots \otimes \xi_{\beta_{d}^{(j)}}
$$

Part b): A formal series $F=\sum_{\alpha} b_{\alpha} H_{\alpha}$ belongs to $(\mathcal{S})^{*}$ if and only if

$$
\sup _{\alpha} b_{\alpha}^{2} \alpha !(2 \mathbf{N})^{-\alpha q}<\infty \text { for some } q<\infty
$$

The action of $F=\sum_{\alpha} b_{\alpha} H_{\alpha} \in(\mathcal{S})^{*}$ on $f=\sum_{\alpha} c_{\alpha} H_{\alpha} \in(\mathcal{S})$ is given by

$$
\langle F, f\rangle=\sum_{\alpha} \alpha ! b_{\alpha} c_{\alpha}
$$

EXAMPLE The pointwise (or singular) white noise $W_{x}$ is defined by

$$
W_{x}(\omega)=\sum_{k=1}^{\infty} e_{k}(x) H_{\epsilon_{k}}(\omega)=\sum_{k=1}^{\infty} e_{k}(x) h_{1}\left(\theta_{k}\right)
$$

where $\epsilon_{k}=(0,0, \cdots, 0,1)$ with 1 on k'th place.

In this case 


$$
\begin{aligned}
& b_{\alpha}=b_{\epsilon_{k}}=e_{k}(x) \text { if } \alpha=\epsilon_{k} \text { for some } k \\
& b_{\alpha}=0 \text { if } \alpha \neq \epsilon_{k} \text { for all } k
\end{aligned}
$$

Moreover, if $\alpha=\epsilon_{k}$ we have

$$
(2 \mathbf{N})^{\alpha}=2^{d} \beta_{1}^{(k)} \cdots \beta_{d}^{(k)}
$$

So in this case we get

$$
\sup _{\alpha} b_{\alpha}^{2} \alpha !(2 \mathbf{N})^{-\alpha q}=\sup _{k} e_{k}^{2}(x)\left(2^{d} \beta_{1}^{(k)} \cdots \beta_{d}^{(k)}\right)^{-q}<\infty
$$

for all $q>0$, since

$$
\sup _{t \in \mathbf{R}}\left|\xi_{k}(t)\right|=0\left(k^{-\frac{1}{12}}\right) \quad([\mathrm{HiP}])
$$

We conclude that $W_{x}(\omega) \in(\mathcal{S})^{*}$.

Note that if $1<p<\infty$ we have

$$
(\mathcal{S}) \subset L^{p}(\mu) \subset(\mathcal{S})^{*}
$$

However,

$$
L^{1}(\mu) \not \subset(\mathcal{S})^{*} \quad \text { (see e.g. [HL ØUZ 1]) }
$$

For our purposes it turns out to be convenient to work with the Kondratiev spaces $(\mathcal{S})^{1}$ and $(\mathcal{S})^{-1}$ which are related to $(\mathcal{S})$ and $(\mathcal{S})^{*}$ as follows:

$$
(\mathcal{S})^{1} \subset(\mathcal{S}) \subset(\mathcal{S})^{*} \subset(\mathcal{S})^{-1}
$$

The spaces $(\mathcal{S})$ and $(\mathcal{S})^{-1}$ were originally constructed on spaces of sequences by Kondratiev $[\mathrm{K}]$ and later extended by him and several other authors. See [KLS] and the references there. We recall here their basic properties, stated in forms which are convenient for our purposes. For details and proofs we refer to [KLS].

\section{DEFINITION 1.2 [KLS]}

Part a): For $0 \leq \rho \leq 1$ let $(\mathcal{S})^{\rho}$ (the Kondratiev space of stochastic test functions) consist of all

$$
\begin{gathered}
f=\sum_{\alpha} c_{\alpha} H_{\alpha} \in L^{2}(\mu) \quad \text { such that } \\
\|f\|_{\rho, k}^{2}:=\sum_{\alpha} c_{\alpha}^{2}(\alpha !)^{1+\rho}(2 \mathbf{N})^{\alpha k}<\infty \quad \text { for all } k<\infty
\end{gathered}
$$

Part b): The Kondratiev space of stochastic distributions, $(\mathcal{S})^{-\rho}$, consists of all formal expansions

$$
F=\sum_{\alpha} b_{\alpha} H_{\alpha}
$$


such that

$$
\sum_{\alpha} b_{\alpha}^{2}(\alpha !)^{1-\rho}(2 \mathbf{N})^{-\alpha q}<\infty \text { for some } q<\infty
$$

The family of seminorms $\|f\|_{\rho, k}^{2} ; k=1,2, \cdots$ gives rise to a topology on $(\mathcal{S})^{\rho}$ and we can then regard $(\mathcal{S})^{-\rho}$ as the dual of $(\mathcal{S})^{\rho}$ by the action

$$
\langle F, f\rangle=\sum_{\alpha} b_{\alpha} c_{\alpha} \alpha !
$$

if $F=\sum b_{\alpha} H_{\alpha} \in(\mathcal{S})^{-\rho}$ and $f=\sum c_{\alpha} H_{\alpha} \in(\mathcal{S})^{\rho}$.

\section{REMARKS.}

1) Regarding (1.21), note that

$$
\begin{aligned}
& \sum_{\alpha}\left|b_{\alpha} c_{\alpha}\right| \alpha !=\sum_{\alpha}\left|b_{\alpha} c_{\alpha}\right|(\alpha !)^{\frac{1-\rho}{2}}(\alpha !)^{\frac{1+\rho}{2}} \cdot(2 \mathbf{N})^{\frac{\alpha k}{2}}(2 \mathbf{N})^{-\frac{\alpha k}{2}} \\
& \leq\left[\sum_{\alpha} b_{\alpha}^{2}(\alpha !)^{1-\rho}(2 \mathbf{N})^{-\alpha k}\right]^{\frac{1}{2}} \cdot\left[\sum_{\alpha} c_{\alpha}^{2}(\alpha !)^{1+\rho}(2 \mathbf{N})^{\alpha k}\right]^{\frac{1}{2}} \\
& <\infty \text { for } k \text { large enough. }
\end{aligned}
$$

2) Putting $\rho=0$ we see by comparing $(1.19),(1.20)$ with $(1.10),(1.13)$ that $(\mathcal{S})=(\mathcal{S})^{0}$ and $(\mathcal{S})^{*}=(\mathcal{S})^{-0}$. So for general $\rho \in[0,1]$ we have

$$
(\mathcal{S})^{1} \subset(\mathcal{S})^{\rho} \subset(\mathcal{S})^{0}=(\mathcal{S}) \subset(\mathcal{S})^{*}=(\mathcal{S})^{-0} \subset(\mathcal{S})^{-\rho} \subset(\mathcal{S})^{-1}
$$

(Observe that with this notation $(\mathcal{S})^{0}$ and $(\mathcal{S})^{-0}$ are different spaces).

\section{DEFINITION 1.3}

The Wick product $F \diamond G$ of two elements

$$
\begin{aligned}
& F=\sum_{\alpha} a_{\alpha} H_{\alpha}, G=\sum_{\beta} b_{\beta} H_{\beta} \text { in }(\mathcal{S})^{-1} \text { is defined by } \\
& F \diamond G=\sum_{\alpha, \beta} a_{\alpha} b_{\beta} H_{\alpha+\beta}
\end{aligned}
$$

From Def. 1.2 we get

\section{LEMMA 1.4}

(i) $F, G \in(\mathcal{S})^{-1} \Rightarrow F \diamond G \in(\mathcal{S})^{-1}$

(ii) $f, g \in(\mathcal{S})^{1} \Rightarrow f \diamond g \in(\mathcal{S})^{1}$ 
The Hermite transform [L $\varnothing \mathrm{U} 1-3]$ has a natural extension to $(\mathcal{S})^{-1}$ :

DEFINITION 1.5 If $F=\sum_{\alpha} b_{\alpha} H_{\alpha} \in(\mathcal{S})^{-1}$ then the Hermite transform of $F, \mathcal{H F}=\tilde{F}$, is defined by

$$
\tilde{F}(z)=\mathcal{H} F(z)=\sum_{\alpha} b_{\alpha} z^{\alpha} \quad \text { (whenever convergent) }
$$

where $z=\left(z_{1}, z_{2}, \cdots\right) \in \mathbf{C}^{\mathbf{N}}$ (the space of all sequences of complex numbers) and

$$
z^{\alpha}=z_{1}^{\alpha_{1}} z_{2}^{\alpha_{2}} \cdots z_{m}^{\alpha_{m}} \text { if } \quad \alpha=\left(\alpha_{1}, \cdots, \alpha_{m}\right) .
$$

If $F \in(\mathcal{S})^{-\rho}$ for $\rho<1$ then it is easy to see that $(\mathcal{H} F)\left(z_{1}, z_{2}, \cdots\right)$ converges for all finite sequences $\left(z_{1}, \cdots, z_{m}\right)$ of complex numbers.

If $F \in(\mathcal{S})^{-1}$, however, we can only obtain convergence of $\mathcal{H} F\left(z_{1}, z_{2}, \cdots\right)$ in a neighbourhood of the origin: We have

$$
\sum_{\alpha}\left|b_{\alpha}\right|\left|z^{\alpha}\right| \leq\left[\sum_{\alpha} b_{\alpha}^{2}(2 \mathbf{N})^{-\alpha q}\right]^{\frac{1}{2}} \cdot\left[\sum_{\alpha}\left|z^{\alpha}\right|^{2}(2 \mathbf{N})^{\alpha q}\right]^{\frac{1}{2}}
$$

where the first factor on the right hand side converges for $q$ large enough. For such a value of $q$ we have convergence of the second factor if

$$
z \in \mathbf{B}_{q}(\delta):=\left\{\zeta=\left(\zeta_{1}, \zeta_{2}, \cdots\right) \in \mathbf{C}^{\mathbf{N}} ; \sum_{\alpha \neq 0}\left|\zeta^{\alpha}\right|^{2}(2 \mathbf{N})^{\alpha q}<\delta^{2}\right\}
$$

for some $\delta<\infty$.

The next result is an immediate consequence of Def. 1.3 and Def. 1.5:

LEMMA 1.6 If $F, G \in(\mathcal{S})^{-1}$ then

$$
\mathcal{H}(F \diamond G)(z)=\mathcal{H} F(z) \cdot \mathcal{H} G(z)
$$

for all $z$ such that $\mathcal{H} F(z)$ and $\mathcal{H} G(z)$ exist.

The topology on $(\mathcal{S})^{-1}$ can conveniently be expressed in terms of Hermite transforms as follows:

\section{LEMMA 1.7 [KLS]}

The following are equivalent

(i) $X_{n} \rightarrow X$ in $(\mathcal{S})^{-1}$

(ii) $\exists \delta>0, q<\infty, M<\infty$ such that

$$
\mathcal{H} X_{n}(z) \rightarrow \mathcal{H} X(z) \text { as } n \rightarrow \infty \quad \text { for } z \in \mathbf{B}_{q}(\delta)
$$


and

$$
\left|\mathcal{H} X_{n}(z)\right| \leq M \quad \text { for all } n=1,2, \cdots ; z \in \mathbf{B}_{q}(\delta)
$$

\section{THEOREM 1.8 [KLS] (Characterization theorem for $(\mathcal{S})^{-1}$ )}

Suppose $g\left(z_{1}, z_{2}, \cdots\right)$ is a bounded analytic function on $\mathbf{B}_{q}(\delta)$ for some $\delta>0, q<\infty$. Then there exists $X \in(\mathcal{S})^{-1}$ such that

$$
\mathcal{H} X=g
$$

From this we deduce the following useful result:

\section{COROLLARY 1.9}

Suppose $g=\mathcal{H} X$ for some $X \in(\mathcal{S})^{-1}$. Let $f$ be an analytic function in a neighbourhood of $\zeta_{0}=g(0)$ in $\mathbf{C}$. Then there exists $Y \in(\mathcal{S})^{-1}$ such that

$$
\mathcal{H} Y=f \circ g
$$

Proof. Let $r>0$ be such that $f$ is bounded analytic on $\left\{\zeta \in \mathbf{C} ;\left|\zeta-\zeta_{0}\right|<r\right\}$. Then choose $\delta>0$ and $q<\infty$ such that the function $z \rightarrow g(z)$ is bounded analytic on $\mathbf{B}_{q}(\delta)$ and such that $\left|g(z)-\zeta_{0}\right|<r$ for $z \in \mathbf{B}_{q}(\delta)$. Then $f \circ g$ is bounded analytic in $\mathbf{B}_{q}(\delta)$, so the result follows from Theorem 1.8 .

\section{EXAMPLE 1.10}

a) Let $X \in(\mathcal{S})^{-1}$. Then $X \diamond X=X^{\diamond 2} \in(\mathcal{S})^{-1}$ and more generally $X^{\diamond n} \in(\mathcal{S})^{-1}$ for all natural numbers $n$. Define the Wick exponential of $X, \operatorname{Exp} X$, by

$$
\operatorname{Exp} X=\sum_{n=0}^{\infty} \frac{1}{n !} X^{\diamond n}
$$

Then by Corollary 1.9 applied to $f(z)=e^{z}$ we see that $\operatorname{Exp} X \in(\mathcal{S})^{-1}$ also.

b) In particular, if we choose $X=W_{x}$ (the singular white noise) then $K_{0}:=\operatorname{Exp} W_{x}$ is in fact in $(\mathcal{S})^{*}$. As suggested in [L $\left.\varnothing \mathrm{U} 1\right],[\mathrm{L} \varnothing \mathrm{U} 3]$ the process $K_{0}(x, \omega)$ is a natural model for stochastic permeability in connection with fluid flow in porous media.

c) Other useful applications of Lemma 1.9 include the Wick logarithm $Y=\log X$, which is defined (in $(\mathcal{S})^{-1}$ ) for all $X \in(\mathcal{S})^{-1}$ with $\tilde{X}(0) \neq 0$. For such $X$ we have

$$
\operatorname{Exp}(\log X)=X
$$

and for all $Z \in(\mathcal{S})^{-1}$ we have

$$
\log (\operatorname{Exp} Z)=Z
$$


d) Similarly we note that the Wick-inverse $X^{\diamond(-1)}$ and more generally the Wick powers $X^{\diamond}(\gamma \in \mathbf{R})$ exist in $(\mathcal{S})^{-1}$ for all $X \in(\mathcal{S})^{-1}$ with $\tilde{X}(0) \neq 0$.

\section{REMARK.}

The connection between the $\mathcal{H}$-transform and the $\mathcal{S}$-transform [HKPS] is

$$
\mathcal{H F}\left(z_{1}, z_{2}, \cdots, z_{m}\right)=(\mathcal{S} F)\left(z_{1} e_{1}+\cdots+z_{m} e_{m}\right)
$$

(see e.g. [L $\varnothing U$ 1])

REMARK. We can define what we could call the generalized expectation of an arbitrary $F \in(\mathcal{S})^{-1}$, in spite of the fact that such an $F$ need not even be in $L^{1}(\mu)$ : If $F_{0} \in L^{p}(\mu)$ for $p>1$ then the action of $F_{0}$ on an element $\psi \in(\mathcal{S})^{1}$ is given by

$$
\left\langle F_{0}, \psi\right\rangle=E\left[F_{0} \psi\right]=\int_{\mathcal{S}^{\prime}} F_{0}(\omega) \psi(\omega) d \mu(\omega)
$$

so if $\psi \equiv 1$ then $\left\langle F_{0}, \psi\right\rangle=\left\langle F_{0}, 1\right\rangle$ gives us the expectation of $F_{0}$. On the other hand, if a general $F \in(\mathcal{S})^{-1}$ has the chaos expansion

$$
F=\sum_{\alpha} b_{\alpha} H_{\alpha}
$$

then by $(1.21)$ we have $\langle F, 1\rangle=b_{0}=\tilde{F}(0)$. Hence we define

$$
\tilde{F}(0)=b_{0}=\langle F, 1\rangle
$$

to be the generalized expectation of $F \in(\mathcal{S})^{-1}$.

From now on we will use the notation

$$
E[F]=E_{\mu}[F]:=\tilde{F}(0)
$$

for the generalized expectation of $F \in(\mathcal{S})^{-1}$. Note that with this definition we have

$$
E[F \diamond G]=E[F] \cdot E[G] \text { for all } F, G \in(\mathcal{S})^{-1} \text {. }
$$

and

$$
E[\operatorname{Exp}(X)]=\exp E[X] \text { for all } X \in(\mathcal{S})^{-1} \text {. }
$$

More generally, from the chaos expansion of $F$ we see that the Hermite transform gives us all the actions

$$
\left\langle F,\langle\cdot, \phi\rangle^{\diamond n}\right\rangle
$$


of $F \in(\mathcal{S})^{-1}$ on $\langle\cdot, \phi\rangle^{\circ n} \in(\mathcal{S})^{1}$. Therefore, although $F$ need not exist as a random variable, it exists as a stochastic distribution: Given a stochastic test function we can compute its associated average.

\section{$(\mathcal{S})^{-1}$ processes}

The Kondratiev space $(\mathcal{S})^{-1}$ of stochastic distributions turns out to be the right space to work in, not just for the Burgers equation discussed in this paper, but for several stochastic (partial or ordinary) differential equations. See e.g. [B 3], [HLØUZ 4]. Therefore, the solution we seek will be a function

$$
u=u(t, x): \mathbf{R}^{1+n} \rightarrow(\mathcal{S})^{-1},
$$

which may be regarded as a (stochastic) distribution valued stochastic process. We call such functions $(\mathcal{S})^{-1}$ processes.

The derivative of an $(\mathcal{S})^{-1}$ process $f(t)$ w.r.t. $t$ at $t=t_{0} \in \mathbf{R}$ will then (if it exists) be the element $\eta=\eta\left(t_{0}\right) \in(\mathcal{S})^{-1}$ with the property that

$$
\frac{f\left(t_{0}+h\right)-f\left(t_{0}\right)}{h} \rightarrow \eta\left(t_{0}\right) \quad \text { in } \quad(\mathcal{S})^{-1} \quad \text { as } \quad h \rightarrow 0
$$

If this holds we write $\eta\left(t_{0}\right)=\frac{d f}{d t}\left(t_{0}\right)$ (or $\frac{\partial f}{\partial t}\left(t_{0}\right)$ ). By the characterization of the topology of $(\mathcal{S})^{-1}$ in terms of the Hermite transform (Lemma 1.7) this is equivalent to

$$
\frac{\tilde{f}\left(t_{0}+h ; z\right)-\tilde{f}\left(t_{0} ; z\right)}{h} \rightarrow \tilde{\eta}\left(t_{0} ; z\right)
$$

pointwise boundedly for $z \in \mathbf{B}_{q}(\delta)$; for some $q<\infty, \delta>0$.

For this it suffices that

$$
\frac{d}{d t} \tilde{f}(t ; z)=\tilde{\eta}(t ; z) \quad \text { for } t=t_{0}
$$

pointwise for each $z \in \mathbf{B}_{q}(\delta)$, if we also have that

$$
t \rightarrow \frac{d}{d t} \tilde{f}(t ; z)
$$

is continuous and uniformly bounded, for $z \in \mathbf{B}_{q}(\delta)$ and $t$ in a neighbourhood of $t_{0}$. For if this holds, we can write

$$
\frac{\tilde{f}\left(t_{0}+h ; z\right)-\tilde{f}\left(t_{0} ; z\right)}{h}=\frac{1}{h} \int_{t_{0}}^{t_{0}+h} \frac{d}{d s} \tilde{f}(s ; z) d s \quad \text { for small } h
$$

and therefore this expression is uniformly bounded for $z \in \mathbf{B}_{q}(\delta)$ as $h \rightarrow 0$. If $\frac{d f}{d t}$ exists and is $t$-continuous, we say that the $(\mathcal{S})^{-1}$ process $f(t)$ is $C^{1}$. A similar notation, $C^{k}$, is 
used for higher order derivatives, $k=2,3, \cdots$ (and for continuity if $k=0$ ) and for several variables, $C^{k_{1}, k_{2}, \cdots, k_{m}}$.

In [HL $\varnothing \mathrm{UZ} 1-2]$ a different kind of solution concept is studied: An $L^{p}$ functional process $(p \geq 1)$ is a map

$$
X: S \times \mathbf{R}^{d} \times \mathcal{S}^{\prime} \rightarrow \mathbf{R}
$$

such that

(i) $x \rightarrow X(\phi, x, \omega)$ is (Borel) measurable for all $\phi \in \mathcal{S}, \omega \in \mathcal{S}^{\prime}$

and

(ii) $\omega \rightarrow X(\phi, x, \omega)$ belongs to $L^{p}(\mu)$ for all $\phi \in \mathcal{S}, x \in \mathbf{R}^{d}$.

Intuitively, $X(\phi, x, \omega)$ is the result of measuring the quantity $X$ using the test function ("window") $\phi$ shifted to the point $x$ and in the "experiment" $\omega$.

EXAMPLE. White noise $W$ may be regarded as an $L^{p}$ functional process (for any $p<\infty$ ) by putting

$$
W(p, x, \omega)=W_{\phi_{x}}(\omega),
$$

where

$$
\phi_{x}(y)=\phi(y-x)
$$

REMARK For $L^{1}$ functional processes the definition of Wick product must be extended, since $L^{1}(\mu)$ is not contained in $(\mathcal{S})^{-1}$. See [HL $\varnothing \mathrm{UZ} 1$ ].

\section{§2. FROM THE STOCHASTIC BURGERS EQUATION TO THE STOCHASTIC HEAT EQUATION.}

This transformation was performed in [HLØUZ 2], but only in the context of functional processes and hence with more assumptions than will be needed within $(\mathcal{S})^{-1}$ :

\section{THEOREM 2.1 (The Wick-Cole-Hopf transformation (I))}

Let $N=N(t, x)$ be an $(\mathcal{S})^{-1}$-valued $C^{0,1}$-process and define

$$
w=-\nabla N \text {. }
$$

Assume that there exists an $(\mathcal{S})^{-1}$-valued $C^{1,3}$-process $X(t, x)$ such that

$$
u=-\nabla X
$$

solves the multidimensional Burgers equation

$$
\left\{\begin{array}{cl}
\frac{\partial u_{k}}{\partial t}+\lambda \sum_{j=1}^{n} u_{j} \diamond \frac{\partial u_{k}}{\partial x_{j}} \quad & =\nu \Delta u_{k}+w_{k}(t, x) ; t>0 ; x \in \mathbf{R}^{n} \\
& u_{k}(0, x)=g_{k}(x) ; 1 \leq k \leq n
\end{array}\right.
$$


Then the Wick-Cole-Hopf transform $Y$ of $u$ defined by

$$
Y:=\operatorname{Exp}\left(\frac{\lambda}{2 \nu} X\right)
$$

solves the stochastic heat equation

$$
\left\{\begin{array}{l}
\frac{\partial Y}{\partial t}=\nu \Delta Y+\frac{\lambda}{2 \nu} Y \diamond[N+C] ; t>0 ; x \in \mathbf{R}^{n} \\
Y(0, x)=\operatorname{Exp}\left(\frac{\lambda}{2 \nu} X(0, x)\right)
\end{array}\right.
$$

for some $t$-continuous $(\mathcal{S})^{-1}$-valued process $C(t)$ (independent of $x$ ).

Proof. The proof in the present $(\mathcal{S})^{-1}$-case follows the proof in [HL $\left.\varnothing \mathrm{UZ} 2\right]$ with only minor modifications. For completeness we give the argument:

Substituting (2.1) and (2.3) in (2.2) we get

$$
-\frac{\partial}{\partial x_{k}}\left(\frac{\partial X}{\partial t}\right)+\lambda \sum_{j} \frac{\partial X}{\partial x_{j}} \diamond \frac{\partial}{\partial x_{j}}\left(\frac{\partial X}{\partial x_{k}}\right)=-\nu \sum_{j} \frac{\partial^{2}}{\partial x_{j}^{2}}\left(\frac{\partial X}{\partial x_{k}}\right)-\frac{\partial N}{\partial x_{k}}
$$

or

$$
\frac{\partial X}{\partial t}=\frac{\lambda}{2} \sum_{j}\left(\frac{\partial X}{\partial x_{j}}\right)^{\diamond 2}+\nu \Delta X+N+C,
$$

where $C=C(t)$ is a $t$-continuous, $x$-independent $(\mathcal{S})^{-1}$-process.

Basic Wick calculus rules give that

$$
\frac{\partial Y}{\partial t}=\frac{\lambda}{2 \nu} Y \diamond \frac{\partial X}{\partial t}
$$

$$
\frac{\partial Y}{\partial x_{j}}=\frac{\lambda}{2 \nu} Y \diamond \frac{\partial X}{\partial x_{j}}
$$

Hence

$$
\begin{aligned}
\Delta Y & =\sum_{j} \frac{\partial}{\partial x_{j}}\left(\frac{\partial Y}{\partial x_{j}}\right)=\sum_{j} \frac{\partial}{\partial x_{j}}\left(\frac{\lambda}{2 \nu} Y \diamond \frac{\partial X}{\partial x_{j}}\right) \\
& =\sum_{j}\left(\frac{\lambda}{2 \nu}\right)^{2} Y \diamond\left(\frac{\partial X}{\partial x_{j}}\right)^{\diamond 2}+\sum_{j} \frac{\lambda}{2 \nu} Y \diamond \frac{\partial^{2} X}{\partial x_{j}^{2}} \\
& =\frac{\lambda}{2 \nu} Y \diamond\left[\frac{\lambda}{2 \nu} \sum_{j}\left(\frac{\partial X}{\partial x_{j}}\right)^{\diamond 2}+\Delta X\right]
\end{aligned}
$$


Now apply (2.7), (2.6) and (2.9) to get

$$
\begin{aligned}
\frac{\partial Y}{\partial t} & =\frac{\lambda}{2 \nu} Y \diamond\left[\frac{\lambda}{2} \sum_{j}\left(\frac{\partial X}{\partial x_{j}}\right)^{\diamond 2}+\nu \Delta X+N+C\right] \\
& =\frac{\lambda}{2 \nu} Y \diamond\left[\frac{\lambda}{2 \nu} \sum_{j}\left(\frac{\partial X}{\partial x_{j}}\right)^{\diamond 2}+\Delta X\right] \nu+\frac{\lambda}{2 \nu} Y \diamond[N+C] \\
& =\nu \Delta Y+\frac{\lambda}{2 \nu} Y \diamond[N+C], \quad \text { as claimed. }
\end{aligned}
$$

\section{$\S 3 .(\mathcal{S})^{-1}$ - SOLUTION OF THE STOCHASTIC HEAT EQUATION}

We now consider the stochastic heat equation (2.4) obtained by performing the Wick-ColeHopf transformation:

\section{THEOREM 3.1}

Suppose that $H(t, x)$ and $f(x)$ are continuous $(\mathcal{S})^{-1}$-processes. Then the stochastic heat equation

$$
\left\{\begin{array}{l}
\frac{\partial Y}{\partial t}=\nu \Delta Y+H \diamond Y ; t>0, x \in \mathbf{R}^{n} \\
Y(0, x)=f(x) ; x \in \mathbf{R}^{n}
\end{array}\right.
$$

has the unique $(\mathcal{S})^{-1}$-solution

$$
Y(t, x)=\hat{E}^{x}\left[f\left(b_{\alpha t}\right) \diamond \operatorname{Exp}\left(\int_{0}^{t} H\left(s, b_{\alpha s}\right) d s\right)\right]
$$

where $\alpha=\sqrt{2 \nu},\left(b_{t}, \hat{P}^{x}\right)$ is standard Brownian motion in $\mathbf{R}^{n}$ and $\hat{E}^{x}$ denotes expectation with respect to $\hat{P}^{x}$.

Proof. Taking Hermite transforms of (3.1) we get the equation

$$
\left\{\begin{array}{l}
\frac{\partial \tilde{Y}}{\partial t}=\nu \Delta \tilde{Y}+\tilde{H} \cdot \tilde{Y} \quad ; t>0, x \in \mathbf{R}^{n}, z \in \mathbf{B}_{q}(\delta) \\
\tilde{Y}(0, x)=\tilde{f}(x) \quad ; x \in \mathbf{R}^{n}, z \in \mathbf{B}_{q}(\delta)
\end{array}\right.
$$

where

$$
\tilde{Y}=\tilde{Y}(t, x)=\tilde{Y}\left(t, x ; z_{1}, z_{2}, \cdots\right) \quad ; \quad z \in \mathbf{B}_{q}(\delta)
$$

denotes the Hermite transform of $Y$ etc. and $\mathbf{B}_{q}(\delta)$ is some neighbourhood of 0 in $\mathbf{C}^{\mathbf{N}}$, as defined in $\S 1$. Fix $z \in \mathbf{B}_{q}(\delta)$. Then by the complex version of the Feynman-Kac formula the solution $\tilde{Y}(t, x, z)$ of (3.3) can be written

$$
\tilde{Y}(t, x ; z)=\hat{E}^{x}\left[\tilde{f}\left(b_{\alpha t} ; z\right) \exp \left[\int_{0}^{t} \tilde{H}\left(s, b_{\alpha s} ; z\right) d s\right]\right]
$$


with $\left(b_{t}, \hat{P}^{x}\right)$ as described above.

This is the Hermite transform of the $(\mathcal{S})^{-1}$ process

$$
Y(t, x)=\hat{E}^{x}\left[f\left(b_{\alpha t}\right) \diamond \operatorname{Exp}\left(\int_{0}^{t} H\left(s, b_{\alpha s}\right) d s\right]\right]
$$

and the proof is complete.

REMARK. The equation

$$
\frac{\partial u}{\partial t}=\Delta u+W \diamond u, \text { where } W \text { is white noise }
$$

has been studied in [NZ]. They prove the existence of a solution of a type they call generalized Wiener functionals.

\section{§4. FROM THE STOCHASTIC HEAT EQUATION TO THE STOCHASTIC BURGERS EQUATION}

Using the fact that an analytic function composed with a Hermite transform (of an $(\mathcal{S})^{-1}$ element) is again the Hermite transform (Corollary 1.9), we can now construct the inverse of the transformation in $\S 2$ :

\section{THEOREM 4.1 (The Wick-Cole-Hopf transformation (II))}

Suppose that $Y(t, x)$ is an $(\mathcal{S})^{-1}$-process which solves the stochastic heat equation

$$
\left\{\begin{array}{l}
\frac{\partial Y}{\partial t}=\nu \Delta Y+H \diamond Y \quad ; \quad t>0, x \in \mathbf{R}^{n} \\
Y(0, x)=f(x) \quad ; \quad x \in \mathbf{R}^{n}
\end{array}\right.
$$

where $f(x), H(t, x)$ are given $(\mathcal{S})^{-1}$-processes, continuously differentiable w.r.t $x, H(t, x)$ continuous w.r.t. $t$ and (see (1.29))

$$
E_{\mu}[f(x)]>0 \text { for all } x \in \mathbf{R}^{n}
$$

Then

$$
u(t, x):=-\frac{2 \nu}{\lambda} \nabla(\log Y(t, x)) \in(\mathcal{S})^{-1} \text { for all } t \geq 0, x \in \mathbf{R}^{n}
$$

where Log denotes "Wick-log" (Example 1.10d)) and the gradient is taken with respect to $x\left(\right.$ in $\left.(\mathcal{S})^{-1}\right)$. 
Moreover, $u=\left(u_{1}, \cdots, u_{n}\right)$ solves the stochastic Burgers equation

$$
\left\{\begin{array}{l}
\frac{\partial u_{k}}{\partial t}+\lambda \sum_{j=1}^{n} u_{j} \diamond \frac{\partial u_{k}}{\partial x_{j}}=\nu \Delta u_{k}+w_{k} ; t>0, x \in \mathbf{R}^{n} \\
u_{k}(0, x)=g_{k}(x) ; x \in \mathbf{R}^{n}
\end{array}\right.
$$

where

$$
w_{k}(t, x)=-\frac{2 \nu}{\lambda} \cdot \frac{\partial H}{\partial x_{k}}(t, x)
$$

and

$$
g_{k}(x)=-\frac{2 \nu}{\lambda} f(x)^{\diamond(-1)} \diamond \frac{\partial f}{\partial x_{k}}(x) ; \quad 1 \leq k \leq n
$$

Proof. By Theorem 3.1 the solution $Y$ of (4.1) is given by

$$
Y(t, x)=\hat{E}^{x}\left[f\left(b_{\alpha t}\right) \diamond \operatorname{Exp}\left(\int_{0}^{t} H\left(s, b_{\alpha s}\right) d s\right)\right] \quad ; \quad \alpha=\sqrt{2 \nu}
$$

Therefore, by (1.30)

$$
E_{\mu}[Y(t, x)]=\hat{E}^{x}\left[E_{\mu}\left[f\left(b_{\alpha t}\right)\right] \cdot \exp \left(\int_{0}^{t} E_{\mu}\left[H\left(s, b_{\alpha s}\right)\right] d s\right)\right]>0
$$

for all $t, x$.

We conclude that the Wick-log of $Y, X=\log Y$, exists in $(\mathcal{S})^{-1}$, by Example 1.10d. We can therefore reverse the argument in the proof of Theorem 2.1:

Put

$$
X(t, x)=\frac{2 \nu}{\lambda} \log Y(t, x)
$$

and

$$
u(t, x)=-\nabla X(t, x) \quad(\text { gradient w.r.t. } x)
$$

Then

$$
Y(t, x)=\operatorname{Exp}\left(\frac{\lambda}{2 \nu} X(t, x)\right)
$$


namely

$$
u(t, x)=-\frac{2 \nu}{\lambda} \nabla \log Y(t, x)
$$

where $Y$ is given by (4.7), i.e.

$$
Y(t, x)=\hat{E}^{x}\left[h\left(b_{\alpha t}\right)^{\diamond \frac{\lambda}{2 \nu}} \diamond \operatorname{Exp}\left(\frac{\lambda}{2 \nu} \int_{0}^{t} N\left(s, b_{\alpha s}\right) d s\right)\right], \quad \alpha=\sqrt{2 \nu} .
$$

b) (Uniqueness): Moreover, this process $u$ in (5.3)-(5.4) is the only solution of (5.2) of gradient form, i.e. which is the gradient w.r.t. $x$ of some continuously $x$-differentiable $(\mathcal{S})^{-1}$ process.

Proof.

a) Apply Theorem 4.1 to the case when

$$
H(t, x)=\frac{\lambda}{2 \nu} N(t, x) \quad, \quad f(x)=h(x)^{\diamond} \frac{\lambda}{2 \nu}
$$

b) If $u$ solves (5.2) and

$$
u(t, x)=-\nabla X(t, x)
$$

then by Theorem 2.1 the process $Y=\operatorname{Exp}\left(\frac{\lambda}{2 \nu} X\right)$ solves (3.1) with $H=\frac{\lambda}{2 \nu}[N+C]$ for some $x$-independent $C(t) \in(\mathcal{S})^{-1}$, and with

$$
f(x)=\operatorname{Exp}\left(\frac{\lambda}{2 \nu} X(0, x)\right)
$$

By Theorem $3.1 Y$ is unique. Then $X=\frac{2 \nu}{\lambda} \log Y$ is unique up to a constant and therefore $u$ is unique. That completes the proof.

\section{Acknowledgements}

We are grateful to Y. Kondratiev and G. Våge for valuable comments.

This work is partially supported by VISTA, a research cooperation between The Norwegian Academy of Science and Letters and Den Norske Stats Oljeselskap A.S. (Statoil).

\section{REFERENCES}

[AKS] S. Albeverio, J. Kondratiev and L. Streit: Spaces of white noise distributions: Constructions, Descriptions, Applications II. Manuscript 1993.

[B 1] F.E. Benth: Integrals in the Hida distribution space $(\mathcal{S})^{*}$. In T. Lindstrøm, B. Øksendal and A. S. Ustunel (editors): Stochastic Analysis and Related Topics. Gordon \& Breach 1993, 89-99. 
[B 2] F.E. Benth: A note on population growth in a crowded stochastic environment. Manuscript, University of Oslo 1993.

[BCJ-L] L. Bertini, N. Cancrini and G. Jona-Lasinio: The stochastic Burgers equation. Manuscript 1993.

[DDT] G. DaPrato, A. Debussche and R. Teman: Stochastic Burgers equation. Preprint, Scuola Normale Superiore Pisa 1993.

[DG] G. DaPrato and D. Gatarek: Stochastic Burgers equation with correlated noise. Preprint, Scuola Normale Superiore Pisa 1994.

[GHL ØUZ] H. Gjessing, H. Holden, T. Lindstrøm, J. Ubøe and T.-S. Zhang: The Wick product. In H. Niemi, G. Högnäs, A.N. Shiryaev and A. Melnikov (editors): "Frontiers in Pure and Applied Probability", Vol. 1. TVP Publishers, Moscow, 1993, pp. 29-67.

[GjHøUZ] J. Gjerde, H. Holden, B. Øksendal, J. Ubøe and T.-S. Zhang: An equation modelling transport of a substance in a stochastic medium. Manuscript 1993.

[GV] I.M. Gelfand and N.Y. Vilenkin: Generalized Functions, Vol. 4: Applications of Harmonic Analysis. Academic Press 1964 (English translation).

[H] T. Hida: Brownian Motion. Springer-Verlag 1980.

[HI] T. Hida and N. Ikeda: Analysis on Hilbert space with reproducing kernel arising from multiple Wiener integral. Proc. Fifth Berkeley Symp. Math.Stat.Probab. II, part 1 (1965), 117-143.

[HKPS] T. Hida, H.-H. Kuo, J. Potthoff and L. Streit: White Noise Analysis. Kluwer 1993.

[HL ØU 1] H. Holden, T. Lindstrøm, B. Øksendal and J. Ubøe: Discrete Wick calculus and stochastic functional equations. Potential Analysis 1 (1992), 291-306.

[HL ØU 2] H. Holden, T. Lindstrøm, B. Øksendal and J. Ubøe: Discrete Wick products. In T. Lindstr $\varnothing \mathrm{m}$, B. $\varnothing \mathrm{ksendal}$ and A.S. Ustunel (editors): Stochastic Analysis and Related Topics. Gordon \& Breach 1993, pp. 123-148.

[HLØUZ 1] H. Holden, T. Lindstrøm, B. Øksendal, J. Ubøe and T.-S. Zhang: Stochastic boundary value problems: A white noise functional approach. Probab. Th. Rel. Fields 95 (1993), 391-419.

[HL ØUZ 2] H. Holden, T. Lindstrøm, B. Øksendal, J. Ubøe and T.-S. Zhang: The Burgers equation with a noisy force. Comm. PDE 19 (1994), 119-141.

[HL ØUZ 3] H. Holden, T. Lindstrøm, B. Øksendal, J. Ubøe and T.-S. Zhang: A comparison experiment for Wick multiplication and ordinary multiplication. In T. Lind- 
strøm, B. Øksendal and A.S. Ustunel (editors): Stochastic Analysis and Related Topics. Gordon and Breach 1993, pp. 149-159.

[HL ØUZ 4] H. Holden, T. Lindstrøm, B. Øksendal, J. Ubøe and T.-S. Zhang: The pressure equation for fluid flow in a stochastic medium. Potential Analysis (to appear).

[HiP] E. Hille and R.S. Phillips: Functional Analysis and Semigroups. Amer. Math. Soc. Colloq. Publ. 31 (1957).

[K] Y. Kondratiev: Generalized functions in problems of infinite dimensional analysis. Ph.D. thesis, Kiev University 1978.

[KLS] Y. Kondratiev, P. Leukert and L. Streit: Wick calculus in Gaussian analysis. Manuscript 1994.

[LøU 1] T. Lindstrøm, B. Øksendal and J. Ubøe: Stochastic differential equations involving positive noise. In M. Barlow and N. Bingham (editors): Stochastic Analysis. Cambridge Univ. Press 1991, pp. 261-303.

[L $\varnothing \mathrm{U}$ 2] T. Lindstrøm, B. Øksendal and J. Ubøe: Wick multiplication and Ito-Skorohod stochastic differential equations. In S. Albeverio et al (editors): Ideas and Methods in Mathematical Analysis, Stochastics, and Applications. Cambridge Univ. Press 1992, pp. 183-206.

[L ØU 3] T. Lindstr $\varnothing \mathrm{m}$, B. $\varnothing \mathrm{ksendal}$ and J. Ubøe: Stochastic modelling of fluid flow in porous media. In S. Chen and J. Yong (editors): Control Theory, Stochastic Analysis and Applications. World Scientific 1991, pp. 156-172.

[NZ] D. Nualart and M. Zakai: Generalized Brownian functionals and the solution to a stochastic partial differential equation. J. Functional Anal. 84 (1989), 279-296.

[Ø] B. Øksendal: Stochastic partial differential equations and applications to hydrodynamics. Submitted to L. Streit (editor): Stochastic Analysis and Applications in Physics (to appear).

[Z] T.-S. Zhang: Characterizations of white noise test functions and Hida distributions. Stochastics 41 (1992), 71-87. 